\title{
The Role of Depression in Chronic Disease Management: An Analysis of the U.S. Behavioral Risk Factor Surveillance System
}

\author{
Bynum $\mathrm{L}^{1,2}$, Kilmer $\mathrm{G}^{3}$, George $\mathbf{M}^{2}$, Eudy $\mathrm{R}^{2}$, Whiteside-Mansell $\mathrm{L}^{2}$ and \\ Balamurugan $\mathrm{A}^{1 *}$ \\ ${ }^{1}$ Arkansas Department of Health, USA \\ 2University of Arkansas for Medical Sciences, USA \\ ${ }^{3}$ Research Triangle Institute, USA
}

Research Article

Volume 2 Issue 1

Received Date: May 22, 2018

Published Date: May 30, 2018

DOI: $10.23880 /$ phoa- 16000124

*Corresponding author: Appathurai Balamurugan, Arkansas Department of Health 4815, W. Markham, Slot 6, Little Rock, Arkansas 72205, USA, Tel: 501-280-4055; Fax: 501-280-4040; Email: appathurai.balamurugan@arkansas.gov

\section{Abstract}

Introduction: Depression is a major comorbidity among people with chronic diseases such as diabetes, hypertension, and asthma. The relationship between depression and chronic disease management is complex and not well understood. The objective of our study was to assess the role of depression in effective chronic disease management.

Methods: The study used data from 491,773 respondents participating in the 2013 Behavioral Risk Factor Surveillance System. Chronic conditions were compared for adults with vs. without a self-reported medical history of depression. The odds of reporting no past year physician-led preventive care (physician follow-up, annual eye exam, A1c checks, and foot exam) and no past year patient self-care practices (medication adherence, blood glucose monitoring and watching or reducing salt intake) among those with depression were calculated. A multivariate logistic regression model was used to account for interaction and confounding effects, and adjusted odds ratios were reported.

Results: A significantly higher proportion of those with vs. without depression had diabetes $(15.1 \%$ vs. $9.2 \%$; $\mathrm{p}=<0.0001$ ), hypertension ( $41.8 \%$ vs. $30.5 \%$; $\mathrm{p}=<0.0001$ ), asthma ( $24.3 \%$ vs. $11.9 \% ; \mathrm{p}=<0.0001$ ), and chronic obstructive pulmonary disease ( $14.3 \%$ vs. $4.7 \%$; $\mathrm{p}=<0.0001)$. Using a multivariate adjusted model, we found adults with diabetes had twice the odds of not having their feet checked by a professional in the past year if they had depression, especially if they were under 44 years of age (OR $=2.0,95 \% \mathrm{CI}, 1.41,2.85$ ).

Conclusion: Adults with depression have a higher prevalence of chronic disease, but are less likely to report physicianled preventive care practices. Screening and effectively managing depression in primary care can improve patient outcomes among those populations and enhanced collaboration with behavioral health care professionals may be needed to improve patients' quality of life and reduce chronic disease management costs.

Keywords: Depression; Chronic Diseases; Diabetes; Hypertension; Asthma 
Abbreviations: HPA: Hypothalamic Pituitary Adrenal; WHO: The World Health Organization; BRFSS: The Behavioral Risk Factor Surveillance System; USPSTF: The U.S. Preventive Services Task Force; EPDS: Edinburgh Postnatal Depression Scale; PHQ: Patient Health Questionnaire; EMR: Electronic Medical Record.

\section{Introduction}

Chronic diseases are long-lasting conditions that require effective ongoing management. These conditions cause a major limitation in activity and quality of life. Chronic diseases can affect a person's ability to perform important activities, restricting their engagement in life and their enjoyment of family and friends [1]. A combination of unhealthy lifestyle choices and an aging population is leading to an epidemic of chronic diseases across the United States. Most individuals have more than one chronic condition. The management of multiple chronic conditions has major cost implications [2].

The burden of chronic diseases in the U.S. is tremendous. One out of every 10 Americans (an estimated 25 million individuals) have at least one chronic condition [3]. The numbers of people with chronic conditions are expected to double in the next twenty-five years [4]. Chronic diseases are responsible for 7 of 10 deaths each year, and treating people with chronic diseases accounts for up to $86 \%$ of our nation's health care costs $[5,6]$. It is the leading cause of death and disability in the U.S [7]. Diseases of the heart, malignant neoplasms (cancers), chronic lower respiratory disease, cerebrovascular diseases, diabetes mellitus, Alzheimer's disease, and kidney disease (nephritis, nephrotic syndrome, and nephrosis) together accounted for $65.8 \%$ of all deaths among U.S. males and $67.2 \%$ of all deaths among U.S. females in 2010 [8].

Millions of people with chronic conditions struggle to manage their symptoms. There are various barriers involved in the delivery of chronic disease management. Traditional methods of healthcare delivery are unsuitable for addressing the current needs and demands among U.S. citizens with chronic diseases. Four of the five most expensive health conditions (based on total health care spending in a given year in the United States) are chronic conditions - heart disease, cancer, mental disorders, and pulmonary conditions [9].

Time constraints during physician visit limits the delivery of preventive services, and in general there is a lack of comprehensive office systems for the management of chronic diseases [10,11]. Current practice guidelines are for only 10 of the most common chronic illnesses which include depression, hypertension, diabetes, arthritis, and asthma which all require more time than primary care physicians have available for patient care overall [12].

Comprehensive chronic disease management has emerged as a new strategy for chronic disease care, but a consistent definition has not been adopted [12]. It is difficult, if not impossible, to measure the exact amount of time a physician should spend managing chronic diseases because of variability among patients in their disease processes, responses to medication, and lifestyle and social issues. Medication nonadherence is a common problem that results in poor patient outcomes \& economic consequences. It may be affected by actions from the patient, the provider and the health care system [13]. There are also non-financial challenges faced by those with multiple chronic conditions, such as learning how to manage fatigue, emotional distress and activity limitations. Care coordination is often the missing link. If care is coordinated, then medical and social service providers bring their respective expertise to bear on each individual's health problems in the most effective and coordinated manner [14].

Depression is a major comorbidity among people with chronic diseases such as diabetes, hypertension, and asthma [15]. Patients with chronic medical illnesses have been found to have two- to threefold higher rates of major depression compared with age- and gender-matched primary care patients [16]. Further, patients diagnosed with depression have been shown to have to be more likely to also have chronic conditions and have shorter life spans than patients without depression [17].

Depression is a leading cause of disability worldwide and is associated with significant morbidity and mortality [18]. It is a very common mental health problem and is associated with a great deal of personal suffering, as well as acting as a significant burden on health services [19]. Chronic stress, by initiating changes in the hypothalamicpituitary-adrenal (HPA) axis and the immune system, acts as a trigger for anxiety and depression. The World Health Organization (WHO) has predicted that by 2030 depressive disorders will have the highest disease burden in developed countries [20]. Managing depression effectively is a major challenge and is associated with considerable costs. These costs stem from various sources including direct medical costs of health care system utilization, nonmedical costs associated with patients' out-of-pocket expenses for treatment services, and costs such as cost productivity related to reduced efficiency at 
work and absenteeism [21]. Diagnosing and managing depression in primary care remains an issue of significant public health concern.

Depression is more prevalent and contributes to morbidity in patients with chronic medical conditions such as diabetes, asthma, and hypertension. It remains an independent risk factor for increased morbidity in patients with chronic disease such as diabetes, asthma and hypertension who experience a greater likelihood of developing depression, which can limit rehabilitation and recovery [22]. Patients with chronic disease such as diabetes, asthma and hypertension and with or without depression, their self-care or preventive care behavior will differ by depression status. Hence, depression is a "confounder" or "identifier" in better control of diabetes [23]. Furthermore, it may be an independent risk factor for increased morbidity in patients with chronic diseases, as it can limit rehabilitation and recovery [24]. Physicianled preventive care and patient self-care practices among patients with chronic diseases such as diabetes, asthma and hypertension may differ by depression status. The research question for this study, "Is depression associated with effective patient and physician -led preventive chronic disease management? "The Centers for Disease Control and Prevention's Behavioral Risk Factor Surveillance system (BRFSS) survey has been used for measuring the prevalence of chronic diseases, health behaviors, and demographic characteristics of the U.S. adult population. The BRFSS system provides publiclyaccessible large data set which allows researchers to address specific queries or conduct specific analyses related to chronic diseases, depression and mental illness. We used the BRFSS survey to evaluate our research question.

\section{Methods}

\section{Data Sources and Study Sample}

The Behavioral Risk Factor Surveillance System (BRFSS): The U.S. BRFSS is the world's largest telephone survey used to track health risks of Americans aged 18 years and older. Since 1984, it has been administered by the 50 states in the US through funding from the Centers for Disease Control and Prevention (CDC) to a random sample of noninstitutionalized community dwelling adults. The survey uses the disproportionate stratified random digit dialing methodology; additional information on survey design and methodology can be found in BRFSS Users Guide (Chapter Seven) [25]. The BRFSS survey collects information on survey respondents' demographic and behavioral characteristics, and co-morbid conditions (presence of diabetes, asthma, hypertension, chronic obstructive pulmonary disease), use of physician-led preventive care (physician follow-up, annual eye exam, A1c checks, and foot exam) and patient self-care practices (medication adherence, blood glucose monitoring and watching or reducing salt intake) among those with and without depression. Data are collected in all 50 states, the District of Columbia, Puerto Rico, the U.S. Virgin Islands, and Guam. The large size of the survey permits calculation of an aggregated nationwide estimate. The core section of the 2013 national BRFSS survey includes one question to assess depression: "Have you ever been told that you have a depressive disorder, including depression, major depression, dysthymia or minor depression?" [26]. Respondents who report yes are defined as having a depressive disorder.

\section{Statistical Analysis}

Statistical analysis was carried out with the SAS version 9.3 (SAS Institute, Cary, North Carolina) to adjust for the complex same design of the national BRFSS survey. A chisquare $\left(X^{2}\right)$ test was used to assess the prevalence of depression by demographics. Point estimates and $\mathrm{p}$ values were calculated to assess the prevalence of depression among those with chronic diseases. A comparison of those with and without depression was analyzed for physician-led preventive care measures (physician follow-up, annual eye exam, A1c checks, and annual foot exam) and patient self-care preventive care measures (medication adherence, blood glucose monitoring and watching or reducing salt intake).When significant interactions were found separate multivariate logistic regression models were examined. A multivariate adjusted model was used to calculate odds ratios for each preventive care measure among those with and without depression.

Point estimates and p-values were calculated to analyze the characteristics (i.e., age group, gender, race, body mass index, income, education, employment, health plan, and current smoker) of among those with and without depression; and to assess the prevalence of depression among those with chronic diseases (i.e., diabetes, hypertension, asthma, and chronic obstructive pulmonary disease).

\section{Results}

The study examined 491,773 people who completed the U.S. Behavioral Risk Factor Survey for survey year 2013. Eighteen percent $(N=95,776)$ of survey respondents were diagnosed with depression. 
The demographic distribution of adults with depression was significantly different from those without depression ( $p<0.0001$ for all characteristics; Table 1). A higher proportion of those with depression were ages 45 to 64 compared to those without depression (40.2\% vs. $33.1 \%)$. Adults with depression were more likely to be female $(64.4 \%$ vs. $48.6 \%)$, more likely to be white $(70.9 \%$ vs. $62.9 \%$ ), and more likely to be unemployed (42.0\% vs. $58.0 \%$ ) compared to those without depression. Also, adults with depression were less likely to have an annual household income of more than $\$ 75,000$ (19.4\% vs. $30.9 \%)$, and less likely to graduate from college $(20.0 \%$ vs. $26.7 \%$ ) compared to those without depression.

Measures related to health behavior and chronic health conditions were different for adults with depression compared to those without. Adults with depression were more likely to report a body mass index (BMI) of 30 or more $(36.9 \%$ vs. $26.4 \%)$, and were more likely to be currently smoke $(30.2 \%$ vs. $15.5 \%)$. Furthermore, a significantly higher proportion of those with depression vs. without had chronic diseases such as diabetes $(15.1 \%$ vs. 9.2\%; $\mathrm{p}=<0.0001$ ), hypertension ( $41.8 \%$ vs. $30.5 \%$; $\mathrm{p}=<0.0001)$, asthma (24.3\% vs. $11.9 \%$; $\mathrm{p}=<0.0001)$, and chronic obstructive pulmonary disease (14.3\% vs. $4.7 \%$; $\mathrm{p}=<0.0001)$.

Associations between depression and physician-led preventive care (physician follow-up, annual eye exam in past year, A1c checks in past year, and annual professional foot exam) and patient self-care practices (high blood pressure medication adherence, daily blood glucose monitoring, watching or reducing salt intake, and daily self-foot exam are shown in Table 2). For physicianled diabetes preventive care, a significantly higher proportion of those with depression reported no past year physician-led preventive care practices such as physician follow-up $(12.3 \%$ vs. $10.6 \%$; $\mathrm{p}=<0.0181)$, annual eye exam in past year $38.2 \%$ vs. $30.6 \%$; $\mathrm{p}=<0.0001)$, A1c test in the past year $(10.8 \%$ vs. $9.0 \%$; $\mathrm{p}=<0.0211$ ), and ages of 45-64 having annual professional foot $\operatorname{exam}(15.9 \%$ vs. 13.3\%; $\mathrm{p}=<0.0031)$. Self-care practices were also associated with depression, and high blood pressure medication nonadherence differed by gender: male (30.1\% vs. $27.1 \% ; \mathrm{p}=<0.0001)$ and female (21.7\% vs.16.3\%; $\mathrm{p}=<0.0001$ ). Furthermore, daily blood glucose monitoring (24.6\% vs. $28.9 \%$; $=<0.0001)$ was associated with depression. There were no differences when assessing the prevalence of physician-led preventive care practices among those ages 18-44 and 65 and older. Similarly, there were no differences in patient self-care for watching/reducing salt intake and practices for daily self-foot exam.
The following physician-led preventive care practices were associated with depression in unadjusted analyses: physician follow-up (OR $=1.18, \mathrm{CI} 1.03,1.36)$, annual dilated eye exam (OR $=1.40, \mathrm{CI} 1.28,1.54)$, and A1c in the past year $(\mathrm{OR}=1.23, \mathrm{CI} 1.03,1.47)$. Annual professional foot examination was also associated with depression, but the magnitude of the odds ratio varied by age group (age 18 - 44: OR = 3.04, CI 2.40, 3.86; age $45-64:$ OR = 2.02, CI $1.79,2.80$; and age $65+:$ OR $=1.39$, CI 1.22, 1.60).

After adjusting for demographic and health behavior characteristics in the multivariate logistic model (Table 3 ), the association remained significant for annual professional foot examination especially among the younger population age 18 to 44 (OR $=2.01$, CI 1.41, 2.85). After adjustment, associations were no longer statistically significant for other physician-led preventive care practices.

The following patient self-care practices were associated with depression in the unadjusted analysis: high blood pressure medication adherence for males (OR $=1.16$, CI 1.06, 1.26) and females (OR = 1.42, CI 1.31, 1.53), daily glucose monitoring ( $O R=1.25$, CI $1.12,1.39$ ), and watching/reducing salt intake was inversely associated $(\mathrm{OR}=0.92$, CI $0.88,0.97$ ). The daily self-foot exam was not statistically significant in the unadjusted analysis.

After adjusting for potential confounders, there was not an association for any of the patient self-care practices. The adjusted model included variables that were significant such as age, gender, race, income, education, health plan, employment status, smoking status, and obesity. Results for annual professional foot examination $(\mathrm{p}=<0.0001)$ were stratified by age and results for high blood pressure medication adherence $(p=<0.005)$ were stratified by gender due to statistically significant interaction effects.

\section{Discussion}

Depression is largely undiagnosed in health care settings. The U.S. Preventive Services Task Force (USPSTF) recommends screening for depression in the general adult population, including pregnant and postpartum women. Screening should be implemented with adequate systems in place to ensure accurate diagnosis, effective treatment, and appropriate follow-up [27]. Self-reported medical history of depression differs among patients with or without chronic diseases such as diabetes, hypertension, asthma, and chronic obstructive pulmonary disease. Prior to adjustment for confounding, 
those with depression had greater odds of reporting no past year physician-led preventive care practices such as physician follow-up, annual eye exam in past year, A1c test in the past year, and annual professional foot exam. Furthermore, adults with depression had greater odds of lack of self-care preventive practices such as high blood pressure medication adherence. Unexpectedly, depression was inversely associated with lack of daily blood glucose monitoring in unadjusted models.

Based on the multiple logistic regression analysis, we found a statistically significant difference whereby individuals adults age 18 to 44 with diabetes had twice the odds of not having their feet checked by a professional if they had depression. Our finding reaffirmed previous studies which identifies concerns about the quality of care of adults with diabetes [28]. Younger adults may have fewer symptoms, and may not seek specialty care. Primary care physicians treating comorbid depression and diabetes should be especially mindful of care guidelines so they can detect problems early and prevent debilitating complications early in life. Also, mental health professionals should encourage patients with chronic conditions to seek quality care on a regular basis, regardless of age.

However, they did include the caveat that it should be done in settings "that have systems in place to assure accurate diagnosis, effective treatment, and follow-up." The USPSTF found adequate evidence that programs combining depression screening with adequate support systems in place improve clinical outcomes (i.e., reduction or remission of depression symptoms) in adults. In addition, the USPSTF found convincing evidence that treatment of adults and older adults with depression identified through screening in primary care settings with antidepressants, psychotherapy, or both decreases clinical morbidity. The USPSTF concludes with at least moderate certainty that there is a moderate net benefit to screening for depression in adults, including older adults, who receive care in clinical practices that have adequate systems in place to ensure accurate diagnosis, effective treatment, and appropriate follow-up after screening [27]. Depression screening program that utilized an electronic medical record (EMR) system has been shown to positively impact and facilitate communication and follow-up. Such programs could help to improve detection and treatment of depression in other primary care settings [29]. Commonly used depression screening instruments include the Patient Health Questionnaire (PHQ) and the Hospital Anxiety and Depression Scales in adults, the Geriatric Depression Scale in older adults, and the Edinburgh Postnatal Depression Scale (EPDS) in postpartum and pregnant women. All positive screening results should lead to additional assessment that considers severity of depression and comorbid psychological problems (eg, anxiety, panic attacks, or substance abuse), alternate diagnoses, and medical conditions. Screening and effectively managing depression in primary care can improve patient outcomes among those with chronic diseases. Physicians should be educated on the importance of collaborating with behavioral health care professionals to improve patient's quality of life and reduce chronic disease management costs.

Many adults in this study were likely misclassified as not having depression because they never visited a health professional and/or were never screened for depression during a visit. This misclassification is possibly differential because adults with chronic conditions may have more health care encounters, each of which is an opportunity to screen for depression. Additionally, adjusted odds ratios often attenuated toward one and were non-significant. This indicates that, while depression is a predictor, other factors associated with depression at least partially explained the greater odds found in the study. Other limitations include a large sample size that could lead to statistically significant but non-meaningful differences in the general population, non-coverage of persons in the military or those residing in institutions, and recall bias. To our knowledge, this is one of the first studies to use the population-based Behavioral Risk Factor Surveillance System (BRFSS) survey data to assess the roles of depression in effective chronic disease management in the U.S. There was a notable difference in physician-led preventive care follow-up among those with and without depression. This study was based on national BRFSS data which included a representative sample size. All 50 states administered the national BRFSS survey whereby inferences can be made to the general U.S. adult population.

\section{Conclusion}

In conclusion, our study demonstrates an association between depression and chronic disease in a random population-based study. Our findings showed that depressed individuals with diabetes were twice as likely to report not having their feet checked by a professional especially if they are between the ages of 18 to 44 . This study provide a support for a team approach in primary care to optimize the care for people with depression and to prevent its' devastating complications related to physician-led annual foot examination. 


\begin{tabular}{|c|c|c|c|}
\hline Characteristics & $\begin{array}{c}\text { With Depression } \\
\left(\%\left(\mathrm{SE}^{*}\right)\right)\end{array}$ & $\begin{array}{c}\text { Without Depression } \\
\left(\%\left(\mathrm{SE}^{*}\right)\right)\end{array}$ & P-value \\
\hline Age groups & & & $<0.0001 \dagger$ \\
\hline $18-44$ & $44.4(0.3)$ & $47.5(0.2)$ & \\
\hline $45-64$ & $40.2(0.3)$ & $33.1(0.2)$ & \\
\hline $65+$ & $15.4(0.2)$ & $19.4(0.1)$ & \\
\hline Gender & & & $<0.0001 \dagger$ \\
\hline Male & $35.6(0.3)$ & $51.4(0.2)$ & \\
\hline Female & $64.4(0.3)$ & $48.6(0.2)$ & \\
\hline Race & & & $<0.0001 \dagger$ \\
\hline White & $70.8(0.3)$ & $62.9(0.2)$ & \\
\hline Black & $9.8(0.2)$ & $12.0(0.1)$ & \\
\hline Other & $19.3(0.3)$ & $25.1(0.2)$ & \\
\hline Income & & & $<0.0001 \dagger$ \\
\hline$<10 \mathrm{~K}$ & $12.6(0.2)$ & $6.0(0.1)$ & \\
\hline $10-24 \mathrm{~K}$ & $32.4(0.3)$ & $22.7(0.2)$ & \\
\hline $25-49 \mathrm{~K}$ & $23.4(0.3)$ & $25.3(0.2)$ & \\
\hline $50-74 \mathrm{~K}$ & $12.2(0.2)$ & $15.2(0.1)$ & \\
\hline$>75 \mathrm{~K}$ & $19.4(0.3)$ & $30.9(0.2)$ & \\
\hline Education & & & $<0.0001 \dagger$ \\
\hline Some high school or less & $19.0(0.3)$ & $14.3(0.1)$ & \\
\hline High school graduate & $28.0(0.3)$ & $28.7(0.1)$ & \\
\hline Some college & $33.1(0.3)$ & $30.3(0.2)$ & \\
\hline College graduate & $20.0(0.2)$ & $26.7(0.1)$ & \\
\hline Employment & & & $<0.0001 \dagger$ \\
\hline Yes & $42.0(0.3)$ & $59.0(0.2)$ & \\
\hline No & $58.0(0.3)$ & $41.0(0.2)$ & \\
\hline Health Plan & & & $<0.0001 \dagger$ \\
\hline Yes & $81.6(0.3)$ & $83.0(0.1)$ & \\
\hline No & $18.4(0.3)$ & $17.0(0.1)$ & \\
\hline Body Mass Index & & & $<0.0001 \dagger$ \\
\hline$<24.9$ & $31.6(0.3)$ & $37.1(0.2)$ & \\
\hline $25.0-29.9$ & $31.5(0.3)$ & $36.4(0.2)$ & \\
\hline$>30.0$ & $36.9(0.3)$ & $26.4(0.2)$ & \\
\hline Current Smoker & & & $<0.0001 \dagger$ \\
\hline Yes & $30.2(0.3)$ & $15.5(0.1)$ & \\
\hline No & $69.8(0.3)$ & $84.5(0.1)$ & \\
\hline Diabetes & & & $<0.0001 \dagger$ \\
\hline Yes & $15.1(0.2)$ & $9.2(0.1)$ & \\
\hline No & $84.9(0.2)$ & $90.8(0.1)$ & \\
\hline Hypertension & & & $<0.0001 \dagger$ \\
\hline Yes & $41.8(0.3)$ & $30.5(0.1)$ & \\
\hline No & $58.2(0.3)$ & $69.5(0.1)$ & \\
\hline Asthma & & & $<0.0001 \dagger$ \\
\hline Yes & $24.3(0.3)$ & $11.9(0.1)$ & \\
\hline No & $75.7(0.3)$ & $88.1(0.1)$ & \\
\hline $\begin{array}{c}\text { Chronic Obstructive Pulmonary } \\
\text { Disease }\end{array}$ & & & $<0.0001 \dagger$ \\
\hline Yes & $14.3(0.2)$ & $4.7(0.1)$ & \\
\hline No & $85.7(0.2)$ & $95.3(0.1)$ & \\
\hline
\end{tabular}

Standard error; † statistically significant.

Table 1: Prevalence Self-reported Medical History of Depression (N=95776) by Demographic Characteristics, National Behavioral Risk Factor Surveillance System, 2013 


\begin{tabular}{|c|c|c|}
\hline Process of Care Measures & At Risk (\% (SE $))$ & P-value \\
\hline \multicolumn{3}{|l|}{ Physician-led Preventive Care Practices } \\
\hline No Physician Follow-up & & $0.0181 \dagger$ \\
\hline With Depression & $12.3(0.7)$ & \\
\hline Without Depression & $10.6(0.4)$ & \\
\hline No Annual dilated eye exam in past year & & $<0.0001 \dagger$ \\
\hline With Depression & $38.2(0.9)$ & \\
\hline Without Depression & $30.6(0.6)$ & \\
\hline No A1c test in the past year & & $0.0211 \dagger$ \\
\hline With Depression & $10.8(0.7)$ & \\
\hline Without Depression & $9.0(0.4)$ & \\
\hline \multicolumn{3}{|l|}{ No Annual professional foot examination* } \\
\hline Age $18-44$ & & 0.0654 \\
\hline With Depression & $20.1(1.7)$ & \\
\hline Without Depression & $16.3(1.2)$ & \\
\hline Age $45-64$ & & $0.0031 \dagger$ \\
\hline With Depression & $15.9(0.7)$ & \\
\hline Without Depression & $13.3(0.5)$ & \\
\hline Age $65+$ & & 0.707 \\
\hline With Depression & $13.1(0.8)$ & \\
\hline Without Depression & $12.8(0.4)$ & \\
\hline \multicolumn{3}{|l|}{ Patient Self-Care Practices } \\
\hline \multicolumn{3}{|l|}{ High blood pressure medication nonadherence* } \\
\hline Male & & $0.0008 \dagger$ \\
\hline With Depression & $30.1(0.8)$ & \\
\hline Without Depression & $27.1(0.4)$ & \\
\hline Female & & $<0.0001 \dagger$ \\
\hline With Depression & $21.7(0.5)$ & \\
\hline Without Depression & $16.3(0.3)$ & \\
\hline No Daily glucose monitoring & & $<0.0001 \dagger$ \\
\hline With Depression & $24.6(0.9)$ & \\
\hline Without Depression & $28.9(0.6)$ & \\
\hline Not Watching/Reducing Salt Intake & & 0.1596 \\
\hline With Depression & $29.8(0.7)$ & \\
\hline Without Depression & $28.6(0.4)$ & \\
\hline No Daily self-foot exam & & 0.8498 \\
\hline With Depression & $36.1(0.9)$ & \\
\hline Without Depression & $35.9(0.6)$ & \\
\hline
\end{tabular}

Table 2: Adults With and Without Depression Reporting No Annual Physician-led Preventive Care Practices andNo Patient Self-Care Practices, National Behavioral Risk Factor Surveillance System, 2013

*Results were stratified because a statistically significant interaction was found between depression and age or gender;

$\sim$ Standard error; $\uparrow$ statistically significant. 


\begin{tabular}{|c|c|c|}
\hline At Risk Characteristics & Unadjusted OR (95\% CI) & Adjusted* OR (95\% CI) \\
\hline \multicolumn{3}{|l|}{ Physician-led Preventive Care Practices } \\
\hline No Physician Follow-up & $1.18(1.03,1.36) \dagger$ & $1.18(0.99,1.42)$ \\
\hline No Annual dilated eye exam in past year & $1.40(1.28,1.54) \dagger$ & $1.09(0.97,1.23)$ \\
\hline No A1c test in the past year & $1.23(1.03,1.47) \dagger$ & $1.14(0.91,1.44)$ \\
\hline \multicolumn{3}{|l|}{ No Annual professional foot examination } \\
\hline \multicolumn{3}{|l|}{ By Age Group } \\
\hline Age 18-44 & $3.04(2.40,3.86) \dagger$ & $2.01(1.41,2.85) \dagger$ \\
\hline Age 45-64 & $2.02(1.79,2.80) \dagger$ & $1.54(1.34,1.77) \dagger$ \\
\hline Age $65+$ & $1.39(1.22,1.60) \dagger$ & $1.20(1.02,1.42) \dagger$ \\
\hline \multicolumn{3}{|l|}{ Patient Self-Care Practices } \\
\hline \multicolumn{3}{|l|}{$\begin{array}{l}\text { High blood pressure medication non } \\
\text { adherence } ~\end{array}$} \\
\hline \multicolumn{3}{|l|}{ By Gender } \\
\hline Male & $1.16(1.06,1.26) \dagger$ & $1.04(0.93,1.17)$ \\
\hline Female & $1.42(1.31,1.53) \dagger$ & $1.10(0.99,1.21)$ \\
\hline No Daily glucose monitoring & $0.80(0.72,0.89) \dagger$ & $0.96(0.85,1.10)$ \\
\hline No Watching/Reducing Salt Intake & $0.92(0.88,0.97) \dagger$ & $1.00(0.95,1.06)$ \\
\hline No Daily self-foot exam & $1.01(0.92,1.11)$ & $1.09(0.96,1.22)$ \\
\hline With Depression & $24.6(0.9)$ & \\
\hline Without Depression & $28.9(0.6)$ & \\
\hline Not Watching/Reducing Salt Intake & & 0.1596 \\
\hline With Depression & $29.8(0.7)$ & \\
\hline Without Depression & $28.6(0.4)$ & \\
\hline No Daily self-foot exam & & 0.8498 \\
\hline With Depression & $36.1(0.9)$ & \\
\hline Without Depression & $35.9(0.6)$ & \\
\hline
\end{tabular}

Table 3: Unadjusted and Adjusted Odds of No Preventive Care Practices for Adults with Depression, National Behavioral Risk Factor Surveillance System, 2013.

*Adjusted for age, gender, race, income, education, health plan, employment status, smoking status, and obesity. $\sim$ Results were stratified because a statistically significant interaction was found between depression and age or gender;

Note: $\mathrm{OR}=$ Odds ratio $\mathrm{CI}=95 \%$ confidence interval $\dagger$ statistically significant.

\section{References}

1. National Council on Aging. (2015) Chronic Disease Self-Management Facts.

2. U.S. Department of Health \& Human Services. (2016) The Challenge of Managing Multiple Chronic Conditions

3. Norris $S$, Glasgow $R$, Engelgau $M$, O'Connor $P$, McCulloch D (2003) Chronic Disease Management: A Definition and Systematic Approach to Component Interventions. Disease Management \& Health Outcomes 11(8): 477-488.

4. Bodenheimer $\mathrm{T}$, Chen $\mathrm{E}$, Bennett $\mathrm{H}$ (2009) Confronting The Growing Burden Of Chronic Disease:
Can The U.S. Health Care Workforce Do The Job? Health Aff 28(1): 64-74.

5. Centers for Disease Control and Prevention, (2015) Chronic Disease Prevention and Health Promotion.

6. (2009) Centers for Disease Control and Prevention. The Power to Prevent, The Call to Control.

7. Bauer UE, Briss PA, Goodman RA, Bowman BA (2014) Prevention of chronic disease in the 21st century: elimination of the leading preventable causes of premature death and disability in the USA. The Lancet 384(9937): 45-52.

8. Heron M (2013) Deaths: leading causes for 2010. Natl Vital Stat Rep 62(6):1-96. 
9. Stanton MW (2006) The High Concentration of U.S. Health Care Expenditures. Research in Action, Issue 19. AHRQ Publication No. 06-0060

10. Committee on Quality Health Care in America, Institute of Medicine (2001) Crossing the Quality Chasm: A New Health System for the 21st Century. Washington, DC: National Academy Press.

11. Yarnall KS, Pollak KI, Ostbye T, Krause KM, Michener JL (2003) Primary care: is there enough time for prevention? Am J Public Health 93(4): 635-641.

12. Ostbye T, Yarnall KS, Krause KM, Pollak KI, Gradison $\mathrm{M}$, et al. (2005). Is there time for management of patients with chronic diseases in primary care? Ann Fam Med 3(3): 209-214.

13. National Conference of State Legislatures (2015) Medication Adherence and Chronic Disease Management.

14. (2016) US Department of Health \& Human Services. The Challenge of Managing Multiple Chronic Conditions

15. Wu S, Green A (2000) Projection of Chronic Illness Prevalence and Cost Inflation Silver Book Reference.

16. Ali S, Stone MA, Peters JL, Davies MJ, Khunti K (2006) The prevalence of comorbid depression in adults with Type 2 diabetes: a systematic review and metaanalysis. Diabet Med 23(11):1165-1173.

17. Zivin K, Pfeiffer PN, Ilgen MA, Welsh DE, McCarthy J, et al. (2012) Early mortality and Years of Potential Life Lost Associated with Depression Among Veterans. Psychiatr Serv 63(8): 823-826.

18. Swartz HA, Rollman BL (2003) Managing the global burden of depression: lessons from the developing world. World Psychiatry 2(3): 162-163.

19. Gilbody S, Bower P (2011) Depression in Primary Care. Cambridge: Cambridge University Press. The Psychiatrist 35(9): p 360.

20. Chambers E, Cook S, Ricketts T, Hutten R, Parry G, et al. (2015) The self-management of longer-term depression: learning from the patient, a qualitative study. BMC Psychiatry 15(1): 1-16.

21. Kooistra L, Wiersma J, Riper H, Lokkerbol J, Cuijpers $\mathrm{P}$, et al. (2014) Blended vs. face-to-face cognitive behavioural treatment for major depression in specialized mental health care: study protocol of a randomized controlled cost-effectiveness trial. BMC Psychiatry 14(1): 1-22.

22. George $M$, Harper $R$, Balamurugan A, Kilmer G, Bynum L, (2011) Diabetic Retinopathy and Its Risk Factors in a Population-Based Study. Journal of primary care \& community health 2(2): 122-126.

23. Lin EH, Katon W, Von Korff M, Rutter C, Simon GE, et al. (2004) Relationship of depression and diabetes self-care, medication adherence, and preventive care. Diabetes care 27(9): 2154-2160.

24. Katon WJ, Lin EH, Von Korff M, Ciechanowski P, Ludman EJ, et al. (2010) Collaborative care for patients with depression and chronic illnesses. New England Journal of Medicine 363(27): 2611-2620.

25. (2006) Behavioral Risk Factor Surveillance System. Operational and User's Guide.

26. (2013) Behavioral Risk Factor Surveillance System. BRFSS Questionnaire.

27. Siu AL, Bibbins-Domingo K, Grossman DC, Baumann LC, Davidson KW, et al. (2016) Screening for Depression in Adults: US Preventive Services Task Force Recommendation Statement. JAMA 315(4): 380-387

28. Egede LE, Ellis C, Grubaugh AL (2009) The effect of depression on self-care behaviors and quality of care in a national sample of adults with diabetes. General hospital psychiatry 31(5): 422-427.

29. Gill JM, Dansky BS (2003) Use of an Electronic Medical Record to Facilitate Screening for Depression in Primary Care. Primary Care Companion to The Journal of Clinical Psychiatry 5(3): 125-128. 